2013

\title{
Atomic Force Microscopy Characterization of Collagen 'Nanostraws' in Human Costal Cartilage
}

\author{
Michael W. Stacey \\ Diganta Dutta \\ Old Dominion University \\ Anthony Asmar \\ Old Dominion University \\ H. Elsayed-Ali \\ Old Dominion University \\ R. Kelly Jr. \\ See next page for additional authors
}

Old Dominion University, mstacey@odu.edu

Follow this and additional works at: https://digitalcommons.odu.edu/bioelectrics_pubs

Part of the Biomedical Engineering and Bioengineering Commons, and the Nanotechnology Commons

\section{Repository Citation}

Stacey, Michael W.; Dutta, Diganta; Asmar, Anthony; Elsayed-Ali, H.; Kelly, R. Jr.; and Beskok, A., "Atomic Force Microscopy Characterization of Collagen 'Nanostraws' in Human Costal Cartilage" (2013). Bioelectrics Publications. 66.

https://digitalcommons.odu.edu/bioelectrics_pubs/66 
Authors

Michael W. Stacey, Diganta Dutta, Anthony Asmar, H. Elsayed-Ali, R. Kelly Jr., and A. Beskok 


\section{Atomic Force Microscopy Characterization of Collagen 'Nanostraws' in Human Costal Cartilage}

Stacey $\mathrm{M}^{1,2}$, Dutta $\mathrm{D}^{3}$, Cao $\mathrm{W}^{4}$, Asmar $\mathrm{A}^{1}$, Elsayed-Ali $\mathrm{H}^{4}$, Kelly Jr R ${ }^{5}$, Beskok $\mathrm{A}^{3}$

${ }^{1}$ Frank Reidy Research Center for Bioelectrics, Old Dominion University, Norfolk, VA

USA

${ }^{2}$ Department of Pediatrics, Eastern Virginia Medical School, Norfolk VA USA

${ }^{3}$ Institute of Micro \& Nanotechnology, Mechanical \& Aerospace Engineering

Department, Old Dominion University, Norfolk, VA USA

${ }^{4}$ Applied Research Center, Electrical and Computer Engineering, Old Dominion University, Norfolk, VA USA

${ }^{5}$ Department of Surgery, Eastern Virginia Medical School and Pediatric Surgery

Division, Children's Hospital of the King's Daughters, Norfolk VA USA

Corresponding author:

Dr. Michael Stacey, Frank Reidy Research Center for Bioelectrics, 4211 Monarch Way, Suite 300, Norfolk, VA 23508 USA

Email: $\underline{\text { mstacey@odu.edu }}$

Telephone: 7576832245

Fax: 7574511010 


\begin{abstract}
Costal cartilage, a type of hyaline cartilage that bridges the bony ribs and sternum, is relatively understudied compared to the load bearing cartilages. Deformities of costal cartilage can result in deformation of the chest wall, , where the sternum is largely pushed towards or away from the spine, pectus excavatum and pectus carinatum respectively, with each condition having significant clinical impact. In the absence of extensive literature describing morphological features of costal cartilage, we characterized a sample from the costal margin immunohistologically and through atomic force microscopy. We had previously observed the presence of collagen 'nanostraws' running the length of costal cartilage. Hypothesizing that these structures may be responsible for fluid flow within this thick, avascular tissue, and prior to microfluidic analysis, we estimated the diameters and measured Young's modulus of elasticity of the collagen nanostraws. We found significant differences in results between treatment type and fixation. Significant differences in nanostraw strength and diameter obviously affect nano-fluidic transport calculations, and, therefore, we consider these results of importance to the scientific community relying upon measurements in the nanoscale.
\end{abstract}




\section{Introduction}

The atomic force microscope (AFM) is a very high resolution scanning probe microscope that has found applications from the biological to the material sciences and has several advantages over transmission and scanning electron microscopy, including the absence of electron-induced specimen damage, ambient operation, preservation of biological morphology, and the ability to be utilized on live or fixed tissues. Analysis of biological samples frequently necessitates their fixation and protein cross-linking by chemical fixation, although fixation itself can cause tissue distortion. The AFM probes the surface topography of a sample to a very high resolution irrespective of whether the tissue is live or fixed. Probing of live tissues opens the possibility of investigating biomechanical measurements, for example, Young's modulus of elasticity [Stolz 2004, Cai 2011, Tripathy and Berger 2012]. Because AFM probing can be undertaken when the sample is submerged, it is possible to maintain live samples under physiological conditions.

Cartilage is a fibrous tissue predominantly consisting of collagens (60-80\% dry weight) [Korhonen and Saarakkala 2011]. Collagen fibers have been extensively studied, and their association with connective tissue disorders described. Recently, AFM has been used to measure differences in collagen fiber diameters in patients with osteoarthritis compared to controls [Stolz 2009]. The arrangement of collagen fibers in costal cartilage has recently been described [Stacey 2012] and shows straw-like structures running the length of the tissue. Highly ordered fiber formation was also described [Forman 2010] in the surrounding perichondrium. Collagen tubules in juvenile rabbit tibia articular cartilage have been reported [Gwynn 2000, Hughes 2005], and although functionally different, ultrastructural similarities may be expected in response to cell maintenance and ultimately collagen fiber deposition. Cartilage is avascular and aneural [Quzzine 2012], and nutrients and gas arrive solely by diffusion to interior cells. We proposed that the straw-like structures observed in costal cartilage act as a means of nutrient and gas transport; additionally they provide biomechanical support [Stacey 2012]. The objectives of this paper are to characterize the diameters and Young's modulus of elasticity of collagen nanostraws by AFM to aide in microfluidic studies of flow in this tissue. To improve access to the collagen nanostraws, it was necessary to liberate them by 
enzymatic digestion and/or homogenization. We found significant differences between treatments and fixation. Significant differences in strength and diameter obviously affect

nano-fluidic transport calculations. Therefore, we consider these results of importance to the scientific community relying upon measurements in the nanoscale.

\section{Materials}

\subsection{Cartilage Sample}

Human costal cartilage was obtained from a patient with pectus carinatum severe enough to warrant surgical repair at the Children's Hospital of the King's Daughters, Norfolk, VA, USA. Informed consent was obtained following IRB approval of the protocol at Eastern Virginia Medical School and Old Dominion University. Costal cartilage samples were collected from ribs 6-8 at surgery, cartilages that fuse and join ribs to the lower sternum as the costal margin. Experiments described here were performed on the round, rod-like, mid-sections of cartilage. All samples were snap frozen in liquid nitrogen and stored at $-80{ }^{\circ} \mathrm{C}$ until use.

\subsection{Immunohistochemistry}

Collagen type II is a major structural protein of cartilage, but its presence warranted confirmation in this tissue type. Frozen cartilage samples were mounted in CRYO-OCT Compound (Tissue-Tek, CA USA) and sections ( $5 \mu \mathrm{m})$ generated using a Microm HM525 cryostat. Sections were fixed in ice-cold acetone. Blocking, incubation with primary and secondary antibodies, and washing were performed following manufacturer's guidelines for each antibody. Tissues were incubated with a mouse monoclonal antibody specific for COL2A1 (sc-52658, Santa Cruz, Santa Cruz, CA). Negative controls were produced using normal mouse IgG included in the ImmunoCruz mouse LSAB staining system (sc-2050, Santa Cruz). Electronic images were captured using a CCD camera through an Olympus BX51 with Metamorph and Image J software.

\subsection{Atomic Force Microscopy (AFM)}

Collagen nanostraws were examined under different conditions (Table 1). Thin sections of undigested cartilage were mounted onto cover slips coated with poly L-lysine. 
Collagen straws were released from small pieces of tissue by brief homogenization (Fisher PowerGen 35 micro homogenizer, Pittsburgh, PA) and/or digestion with $1 \mathrm{mg} / \mathrm{ml}$ hyaluronidase and $1 \mathrm{mg} / \mathrm{ml}$ trypsin (Sigma Aldrich) in Sorenson's buffer (BioRad) at 37 ${ }^{\circ} \mathrm{C}$ for 48 hours [Stolz 2009]. Samples were mounted onto microscope cover glass for AFM analysis. AFM imaging was performed in both air and buffer (phosphate buffered saline, $\mathrm{pH} 7.0$ ) at room temperature. Fixed samples were dried and fixed in ice-cold methanol:acetone. All AFM experiments were carried using a calibrated Nanonics Multiview-4000 multi-probe AFM. Calibration for topology measurements was performed using a microfabricated silicon grid provided by the manufacturer, whose dimensions were further verified using a profilometer. Post processing for the AFM force measurements were based on the methods described in [Sader 2004], and verified by obtaining the Young's modulus of elasticity of silicon and polydimethylsiloxane (PDMS). The latter is a polymeric soft material, whose modulus of elasticity was compared with the data reported in [Armani 1999]. Experiments were run in tapping mode using a $20 \mathrm{~nm}$ parabolic quartz tip. Images were acquired using NWS Version 2.3 Software, filtered by the WSxM 5.0 version 3.1 software, and fiber diameters were measured.

\subsection{Scanning Electron Microscopy (SEM)}

Characteristic D-zone bands of collagen were investigated by SEM. Samples were cut into small sections approximately $3 \mathrm{~mm}$ thick, washed three times in Sorenson's buffer, and digested at $37{ }^{\circ} \mathrm{C}$ for 48 hours in trypsin $(1 \mathrm{mg} / \mathrm{ml})$ and hyaluronidase $(1 \mathrm{mg} / \mathrm{ml})$ in Sorenson's buffer. Samples were washed thoroughly in PBS and fixed in $2.5 \%$ glutaraldehyde in phosphate buffered saline for 2.5 hours. Samples were rinsed in deionized water, dehydrated through an ethanol series, dried and mounted on carbon discs, gold sputtered to eliminate charge build-up during SEM analysis, and examined using a calibrated JSM-6060LV SEM (JEOL, Tokyo, Japan) operating at $20 \mathrm{keV}$. Dzones were measured by comparing size bar to ten consecutive D-zones using image-J software (NIH).

\subsection{Statistics}


Statistical analysis was performed using t-test to determine significance between sample and control means. For all tests, $p<0.05$ indicated the difference as significant.

\section{Results and Discussion}

\subsection{COL2A1 presence in costal cartilage}

The presence of COL2A1 in costal cartilage is shown in Figure 1. Staining appears to be intense surrounding cellular lacunae, with a more uniform staining pattern within the matrix. This staining is consistent with COL2A1 staining in hyaline cartilage. Negative controls showed no staining (data not shown).

\subsection{AFM}

Estimated values of nanostraw diameter were smaller for AFM compared to published SEM [Stacey 2012]. AFM samples were exposed to different conditions, including dehydration, homogenization, and fixation processes, all of which might be causing the observed differences in diameter. Indeed, because cartilage consists predominantly of water ( 60\%), initial freezing of samples in liquid nitrogen after surgery may have introduced ice crystals in samples resulting in formation of tubules. Gwynn 2000 explored this possibility in rabbit tibia and concluded the relatively smooth appearance and consistent size of tubules could not be explained by accumulation of large numbers of small ice crystals in the lumen. The fact that we can separate tubules agrees with his observations that these are genuine structures. Table 1 shows nanostraw dimensions measured by AFM. There appear to be significant differences in dimensions dependent upon treatment procedures. Interestingly, the smallest diameters were measured from unfixed samples measured in air or physiological buffer, with no significant difference in size $(p<0.280)$ between the two conditions. Samples unfixed in air were mounted quickly and may not have dried significantly, resulting in comparable values. However, unfixed samples in buffer, where some swelling from hydration forces may be expected [Heim 2007], were not observed. There were significant differences between undigested and enzymatically digested samples $(\mathrm{p}<0.032)$ but homogenization prior to digestion and fixation showed a significant increase in diameter $(\mathrm{p}<0.001)$. Although brief homogenization would be expected to break up the tissue and allow more efficient 
enzymatic digestion, the increase in diameter was unexpected and is likely due to exposure of unfixed tubules to enzymatic digestion. Enzymatic digestion removes proteoglycans that account for $\sim 30 \%$ of cartilage proteins. Although proteoglycans attract water, their removal may also alter physical properties between fibers allowing greater expansion. A comparison of exposure to Sorenson's buffer without enzymatic solution was not made.

Standard deviation (SD) as a percentage of mean diameter (Table 1) shows that undigested and digested alone had the smallest variation around the mean diameter (15\% and $18 \%$, respectively). Homogenized and digested samples had the greatest variation, with unfixed samples showing greatest at $37 \%$, indicating that the process of homogenization may influence nanostraw structure, or more likely result in damaged fibers accounting for the larger variation in size. Interestingly, variation in the structure of costal cartilage was suggested [Tripathy and Berger 2012] where, using AFM, they observed large variation in Young's modulus (2.17-5.49 MPa) over the tissue. Although their analysis was on transverse sections from samples derived from older individuals (31-62 years), ultrastructural analysis presented here on longitudinal straw-like structures provides additional data for interpreting the biomechanical properties of costal cartilage.

D-Zone bands are characteristic of collagen fibers, reflect the underlying regular arrangement of fibrils, and are estimated to be approximately $67 \mathrm{~nm}$ in hydrated and 64 $\mathrm{nm}$ in dehydrated samples [Orgel 2011]. We measured D-Zone patterns from an SEM image compared to a digested and homogenized AFM image in air (Figures 2A and B, respectively) and found mean D-Zone values of $63 \mathrm{~nm}$ and $65 \mathrm{~nm}$ from 10 zones each respectively for SEM and AFM. These results are consistent with shorter D-Zones in dehydrated collagen forms. Much of the controversy surrounding the structure of articular cartilage originally derived from methods used for fixation of the tissue prior to imaging. Different chemical preparation methods produce different results [Kääb 1999]. Chemically, glutaraldehyde has been used extensively as a fixative for electron microscopy. Gwynn 2000 has shown that cartilage fixed in 2.5\% glutaraldehyde shows considerable shrinkage. The slightly smaller D-zone values in the dehydrated SEM images may also be accounted for by the fixation procedure. 
Figure 3 shows topography of various costal cartilage samples. Measurements on nanostraws are improved by digestion and homogenization. The orderly arrangement of collagen fibers can be seen after enzymatic digestion (Figure 3B) compared to an undigested sample (Figure 3A). Individual nanostraws are dislodged following brief homogenization (Figures 3C-E). Smaller individual fibrils of approximately 90-130 nm are also present in these images and may represent collagen fibers dislodged by treatment.

Figure 4 shows typical force measurement on nanostraws for digested, homogenized and fixed specimen both in air (Figure 4A) and PBS buffer (Figure 4B). For measurements in air, utilizing the DMT model [Israelachvili, 2011] the modulus of elasticity from six separate measurements is found to be $2.01 \pm 0.23 \mathrm{GPa}$.. In PBS buffer, we utilized five different measurements and found collagen nanostraws' modulus of elasticity to be $23 \pm 3 \mathrm{MPa}$ using the DMT model. Collagen nanostraws are structures significantly larger than individual collagen fibers and may be cross-linked by numerous proteins. Force measurements in the literature on individual collagen fibers from different species, sources and under different conditions range from $2 \mathrm{MPa}-11 \mathrm{GPa}$ [Heim et al., 2006; Heim et al., 2007; Wenger et al., 2007 Grant et al. 2009]. Here we report on dimensions and Young's modulus of a cartilaginous sub-structure likely to confer important biomechanical properties to this tissue type which are not directly comparable to data from individual collagen fibers.

\section{Conclusions}

Overall, our results show the unusual tubular network of costal cartilage that is hypothesized to act as a means of fluid and gas transport. To study nano-fluidic transport, such structures necessitate the accurate measurements of their dimensions. Interestingly, previous reports suggest that in rabbit tibia these structures correspond to the known biomechanical properties of the tissue, and would act as a dampening system during compression by resisting lateral fluid flow in the tissue and directing it against the compressive force [Gwynn 2002]. Our study demonstrates that the protocols adopted for these measurements have significant influence on size measurement. Clearly, costal cartilage has large fiber dimensions with complex structures that are formed through 
finely tuned fibrillogenesis that ultimately reflect the biology of this understudied tissue type.

\section{Acknowledgements}

This work was supported by NSF CBET-0922809. We acknowledge support from Old

Dominion University Research Foundation for a seed grant.

\section{References}

Armani D, Liu C, Aluru, N. (1999). Re-configurable fluid circuits by PDMS elastomer micromachining. In Proceedings of MEMS '99; Twelfth IEEE International Conference on Micro Electro Mechanical Systems, pp 222-227.

Cai X, You P, Cai J, Yang X, Chen Q, Huang F. (2011). ART-induced biophysical and biochemical alterations of jurkat cell membrane. Micron 42; 17-28

Forman JL, del Pozo de Dios E, Dalmases CA, Kent RW. (2010). The contribution of the perichondrium to the structural mechanical behavior of the costal-cartilage. J. Biomech. Eng. 132; /094501 doi: 10.1115/1.4001976

Grant CA, Brockwell DJ, Radford SE, Thomson NH. (2009). Tuning the elastic modulus of hydrated collagen fibrils. Biophysical J. 97; 2985-2992

Gwynn I ap, Wade S, Kääb MJ, Owen GR, Richards RG. (2000). Freeze-substitution of rabbit tibial articular cartilage reveals that radial zone collagen fibers are tubules. J.

Microsc. 197; 159-172

Gwynn I ap, Wade S, Ito K, Richards RG. (2002). Novel aspects to the structure of rabbit articular cartilage. Eur Cell Mater 2: 18-29.

Heim AJ, Mathews WG, Koob TJ. (2006) Determination of the elastic modulus of native collagen fibrils via radial indentation. Applied Physics Letters 89:181902-181904.

Heim AJ, Koob TJ, Mathews WG. (2007) Low strain nano-mechanics of collagen fibrils. Biomacromolecules 8:3298-3301

Hughes LC, Archer CW, Gwynn I AP. (2005). The ultrastructure of mouse articular cartilage: Collagen orientation and implications for tissue functionality. A polarized light and scanning electron microscope study and review. European Cells and Materials 9; 6884

Israelachvili JN. Intermolecular and Surface Forces. (2011). Chapter 12. Academic Press London. 
Kääb M J, Richards R G, Walther P, ap Gwynn I, Nötzli H P (1999) A Comparison of four preparation methods for the morphological study of articular cartilage for scanning electron microscopy. Scanning Microscopy 13: 61-70.

Korhonen RK and Saarakkala S. (2011) Biomechanics and modeling of skeletal soft tissues. In Theoretical Biomechanics Ed. Vaclav Klika. InTech pp 113-132

Orgel JPRO, San Antonio JD, Antipova O. (2011). Molecular and structural mapping of collagen fibril interactions. Connective Tissue Res. 52; 2-17

Quzzine M, Venkatesan N, Fournel-Gigleux S. (2012) Proteoglycans and cartilage repair. Methods Mol. Biol. 836; 339-355

Sader JE, Jarvis SP. (2004). Accurate formulas for interaction force and energy in frequency modulation force spectroscopy. Applied Physics Letters, 84(10) 1801-1803

Stacey MW, Grubb J, Asmar A, Pryor J, Elsayed-Ali H, Cao W, Beskok A, Dutta D, Darby DA, Fecteau A, Werner A, Kelly RE Jr. Decorin Expression, Straw-like Structure, and Differentiation of Human Costal Cartilage. (2012). Connective Tissue Research. In press

Stolz M, Raiteri R, Daniels AU, VanLandingham MR, Baschong W, Aebi U. (2004). Dynamic elastic modulus of porcine articular cartilage determined at two different levels of tissue organization by indentation type atomic force microscopy. Biophysical Journal $86 ; 3269-3283$

Stolz M, Gottardi R, Raiteri R, Miot S, Martin I, Imer R, Staufer U, Raducanu A, Düggelin M, Baschong W, Daniels AU, Friederich NF, Aszodi A \& Aebi U. (2009). Early detection of aging cartilage and osteoarthritis in mice and patient samples using atomic force microscopy. Nature Nanotechnology 4; 186-192

Tripathy S and Berger EJ (2012). Quasi-linear viscoelastic properties of costal cartilage using atomic force microscopy. Comput. Methods Biomech. Biomed. Engin. 15; 475-486

Wenger MP, Bozec EL, Horton MA, Mesquida P. (2007) Mechanical properties of collagen fibrils. Biophysics J., 93:1255-1263. 


\begin{tabular}{|l|c|c|c|c|c|c|}
\hline & $\begin{array}{c}\text { Undiges } \\
\text { ted } \\
\text { Fixed } \\
\text { Air } \\
(n=74)\end{array}$ & $\begin{array}{c}\text { Digest } \\
\text { ed } \\
\text { Fixed } \\
\text { Air } \\
(n=61)\end{array}$ & $\begin{array}{c}\text { Homogeni } \\
\text { zed } \\
\text { Digested } \\
\text { Fixed } \\
\text { Air (n=61) }\end{array}$ & $\begin{array}{c}\text { Homogeni } \\
\text { zed } \\
\text { Digested } \\
\text { Unfixed } \\
\text { Air }(n=58)\end{array}$ & $\begin{array}{c}\text { Homogeni } \\
\text { zed } \\
\text { Digested } \\
\text { Fixed } \\
\text { Buffer } \\
(n=26)\end{array}$ & $\begin{array}{c}\text { Homogeni } \\
\text { zed } \\
\text { Digested } \\
\text { Unfixed } \\
\text { Buffer } \\
(n=16)\end{array}$ \\
\hline $\begin{array}{l}\text { Mean } \\
\text { Diame } \\
\text { ter +/- } \\
\text { SD } \\
(n m)\end{array}$ & $435+/-64$ & $463+/-$ & $528+/-130$ & $361+/-134$ & $410+/-120$ & $331+/-84$ \\
\hline
\end{tabular}

Table 1. Mean collagen nanostraw diameter (+/- Standard Deviation, SD) under specified conditions of digestion, homogenization, and fixation. AFM measurements were made under air or physiological buffer. 


$$
a^{20}
$$


Figure 2
Click here to download high resolution image
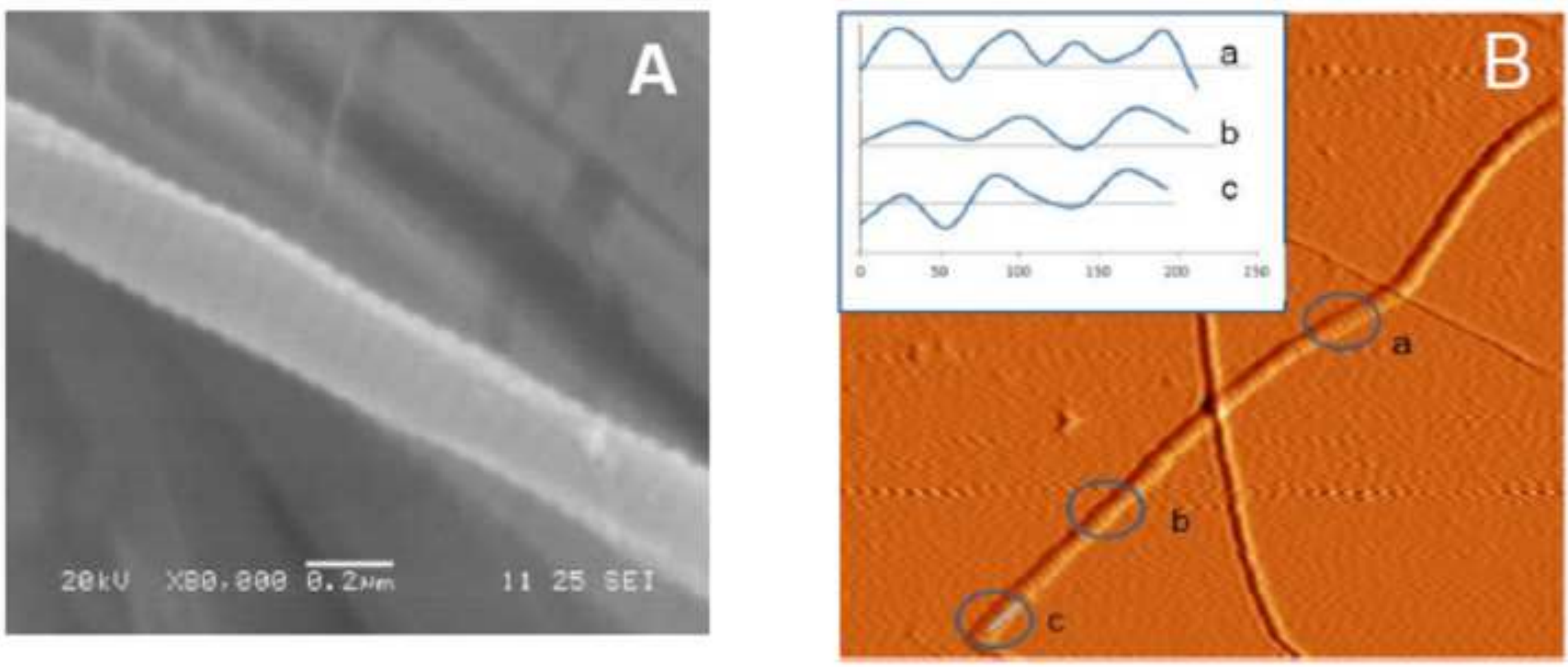
Click here to download high resolution image
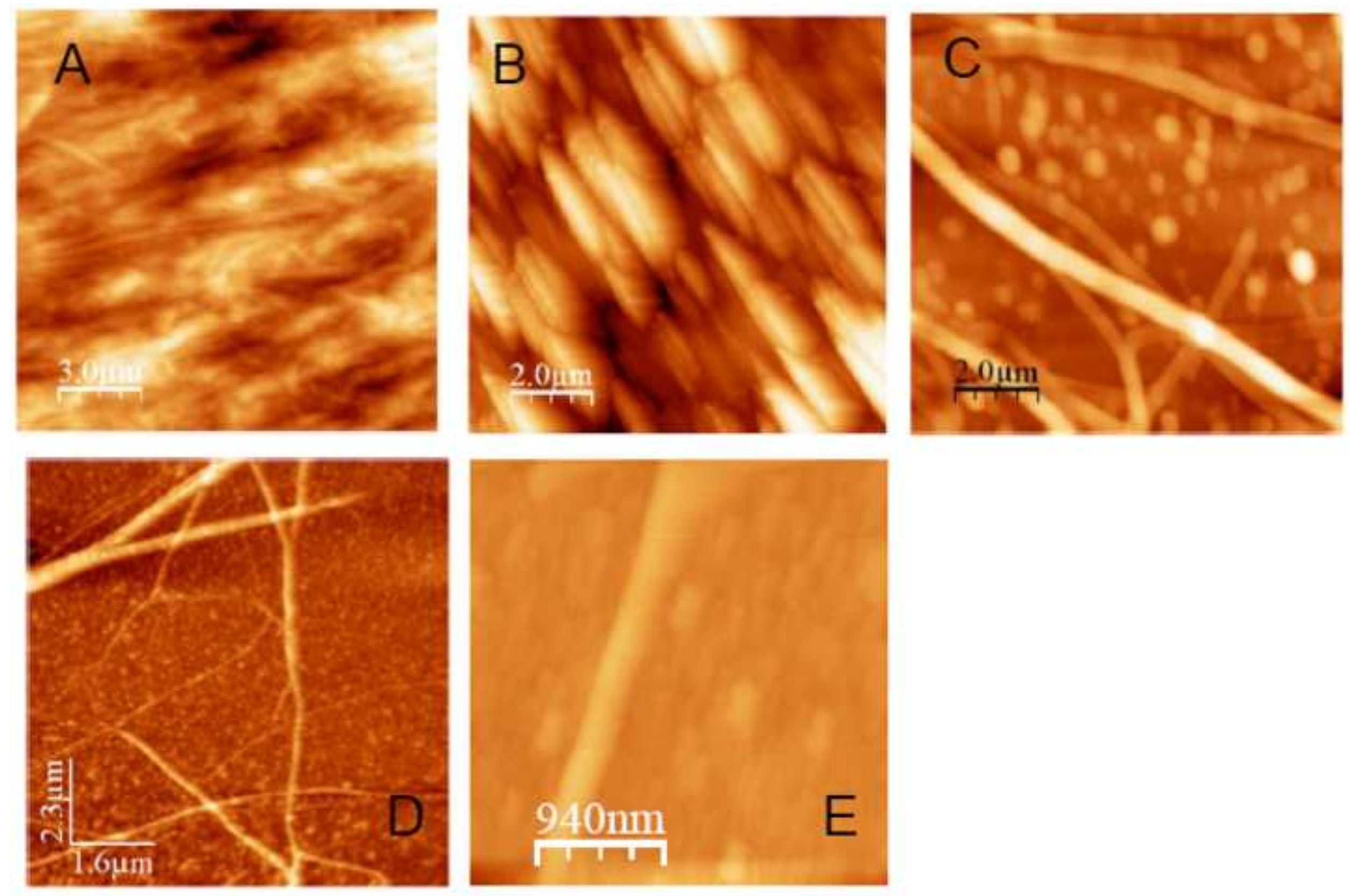


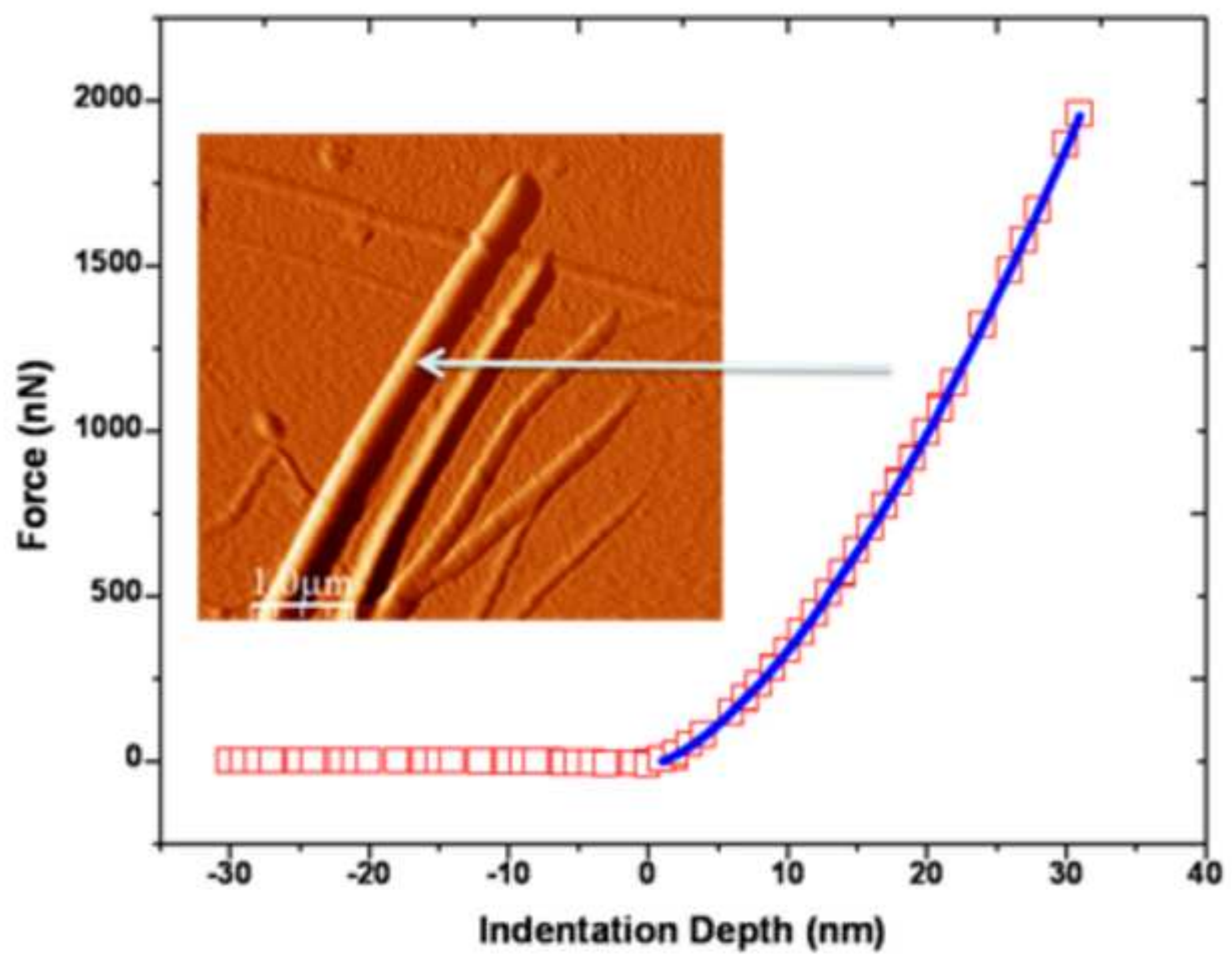


